\title{
Battery Characterization via Eddy-Current Imaging with Nitrogen-Vacancy Centers in Diamond
}

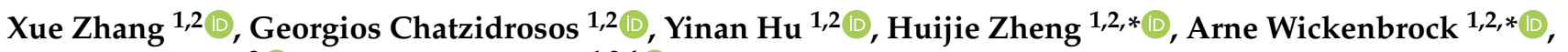 \\ Alexej Jerschow ${ }^{3(D)}$ and Dmitry Budker $1,2,4(\mathbb{D}$ \\ 1 Department of Physics, Johannes Gutenberg-Universität Mainz, 55128 Mainz, Germany; \\ xuezhang@uni-mainz.de (X.Z.); gechatzi@uni-mainz.de (G.C.); yinanhu1@uni-mainz.de (Y.H.); \\ budker@uni-mainz.de (D.B.) \\ 2 Helmholtz-Institut, GSI Helmholtzzentrum für Schwerionenforschung, 55128 Mainz, Germany \\ 3 Department of Chemistry, New York University, New York, NY 10003, USA; alexej.jerschow@nyu.edu \\ 4 Department of Physics, University of California, Berkeley, CA 94720, USA \\ * Correspondence: zheng@uni-mainz.de (H.Z.); wickenbr@uni-mainz.de (A.W.)
}

check for updates

Citation: Zhang, X.; Chatzidrosos, G.; Hu, Y.; Zheng, H.; Wickenbrock, A.; Jerschow, A.; Budker, D. Battery Characterization via Eddy-Current Imaging with Nitrogen-Vacancy Centers in Diamond. Appl. Sci. 2021, 11, 3069. https://doi.org/10.3390/ app11073069

Academic Editor:

Alexander Chroneos

Received: 3 March 2021

Accepted: 26 March 2021

Published: 30 March 2021

Publisher's Note: MDPI stays neutral with regard to jurisdictional claims in published maps and institutional affiliations.

Copyright: (C) 2021 by the authors. Licensee MDPI, Basel, Switzerland. This article is an open access article distributed under the terms and conditions of the Creative Commons Attribution (CC BY) license (https:/ / creativecommons.org/licenses/by/ $4.0 /)$.

\begin{abstract}
Sensitive and accurate diagnostic technologies with magnetic sensors are of great importance for identifying and localizing defects of rechargeable solid batteries using noninvasive detection. We demonstrate a microwave-free alternating current (AC) magnetometry method with negatively charged NV centers in diamond based on a cross-relaxation feature between nitrogenvacancy (NV) centers and individual substitutional nitrogen (P1) centers occurring at $51.2 \mathrm{mT}$. We apply the technique to non-destructively image solid-state batteries. By detecting the eddy-currentinduced magnetic field of the battery, we distinguish a defect on the external electrode and identify structural anomalies within the battery body. The achieved spatial resolution is $360 \mu \mathrm{m}$. The maximum magnetic field and phase shift generated by the battery at the modulation frequency of $5 \mathrm{kHz}$ are estimated as $0.04 \mathrm{mT}$ and 0.03 rad respectively.
\end{abstract}

Keywords: battery diagnostics; NV-centers in diamond; eddy current imaging; nondestructive evaluation

\section{Introduction}

Sensitive and accurate diagnostic technologies with magnetic sensors are of great importance for identifying and localizing defects of rechargeable solid batteries using noninvasive detection. Negatively charged nitrogen-vacancy (NV) centers have been extensively exploited as precise nanoscale probes in applications such as measurement of magnetic fields [1], temperature [2], strain [3], rotation [4,5], electric fields [6,7] and so forth. Recently, NV centers have also been utilized for eddy-current imaging. Eddy-current detection was demonstrated with vapor-cell magnetometers [8-10], and later also with NV diamond [11]. Compared with other sensors, diamond-based devices can be used over a wide temperature range, can have nanoscale spatial resolution, high sensitivity and wide bandwidth. In [11], a microwave-free eddy-current imaging device based on NV centers in diamond was demonstrated making use of a NV-NV cross-relaxation feature between 0 and $20 \mathrm{mT}$. In this paper, we report imaging based on a much narrower cross-relaxation feature between the NV centers and substitutional nitrogen (P1) centers and apply it to perform non-destructive evaluation experiments on small solid-state batteries.

The imaged sample is an all-ceramic multilayer solid-state battery produced by TDK Corporation. It incorporates inner electrodes, electrolyte and external electrodes. When the battery is placed in an oscillating magnetic field (primary field), eddy currents flow in the electrodes and the electrolyte, which, in turn, in our case induce an alternating magnetic field (secondary field).

We demonstrate all-optical AC magnetometry to detect the secondary field as a function of position. The secondary field is anti-parallel to the primary field and exhibits a 
phase delay. These quantities relate to a number of characteristics in the imaged sample, such as shape, dimensions, conductivity and susceptibility. As a result, one can use NV centers as probes to distinguish textures and identify structural anomalies inside and outside the battery.

\section{Experimental Apparatus}

A schematic of the experimental setup is shown in Figure 1. Linearly polarized light from a continuous-wave green laser at $532 \mathrm{~nm}$ is continuously pumping the NV centers in a diamond sample. The laser power can be adjusted with a polarizer and a half-waveplate. NV centers in the excited state decay back to the ground state both via radiative and nonradiative transitions, with red photoluminescence (PL) emitted in the former case. The fact that NV centers in the $m_{s}= \pm 1$ magnetic sublevels have significantly higher probability to undergo an intersystem crossing (ISC) [12], i.e., a nonradiative transition from the triplet to the singlet excited state, results in the majority of NV centers being optically pumped into the $m_{s}=0$ spin projection. In order to minimize the noise caused by intensity fluctuations in the laser beam, we use a light-power stabilization loop incorporating a photodiode (PD2), a proportional-integral-differential (PID) controller, and an acousto-optic modulator (AOM).

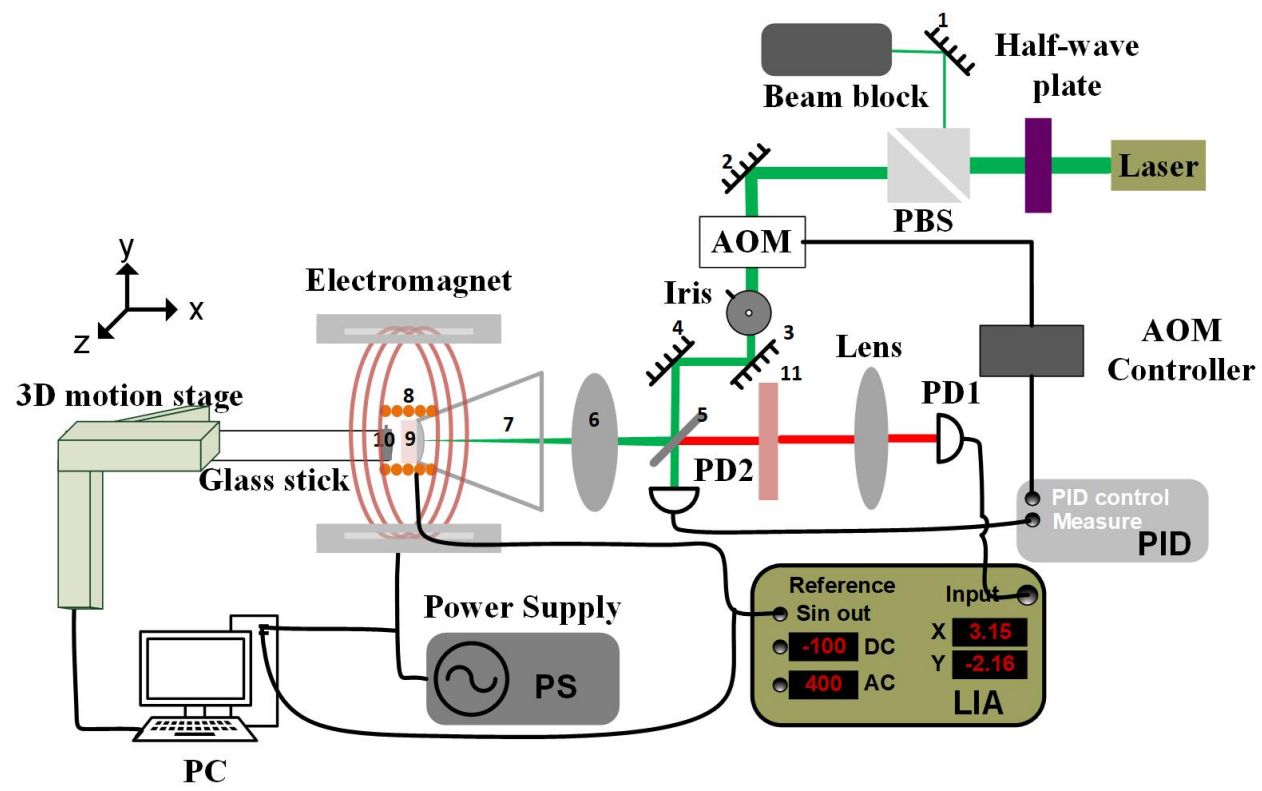

Figure 1. Experimental setup. 1-4: mirrors; 5: dichroic mirror; 6: lens; 7: parabolic concentrator; 8: RF coil; 9: diamond; 10: battery; 11: notch filter. PBS: polarizing beamsplitter; AOM: acousto-optic modulator; PD1,2: photodiodes; PC: personal computer; PS: power supply.

A dichroic mirror and a notch filter are used to filter out red PL from the green light. The diamond used in this setup is a type-Ib, (111)-cut, high-pressure-high-temperaturegrown sample with dimensions $3.0 \times 3.0 \times 0.4 \mathrm{~mm}^{3}$. The initial nitrogen concentration was specified as less than $110 \mathrm{ppm}$. Then nitrogen-rich sample was electron-irradiated and annealed [11]. The diamond is glued on the top plane of a parabolic concentrator used to collect fluorescence. A radio-frequency (RF) coil is made from copper wire of $0.1 \mathrm{~mm}$ diameter. It has five turns and is wound around the diamond to provide a modulation field to both the diamond and battery. The modulation field is provided by the lock-in amplifier (LIA) used for the AC magnetometric scheme. The frequency of the field (maximum $4 \mathrm{MHz}$ ) is also used as the LIA reference for detection.

A 3D translation stage is used to fast-scan the battery in front of the diamond. The battery is glued on top of a $24 \mathrm{~cm}$ long polyvinyl chloride (PVC) rod attached to a motorized three-dimension translation stage controlled by a computer. A variable background field is 
provided by a custom-made electromagnet (EM). This field is required for the microwavefree magnetic field detection method. The EM consists of approximately 200 turns wound with a rectangular cross-section $\left(1.4 \times 0.8 \mathrm{~mm}^{2}\right)$ wire around a $5 \mathrm{~cm}$-diameter bore. The coil is wound on a water-cooled copper mount, and produces a background field of $2.9 \mathrm{mT}$ per ampere supplied. Diamond, battery and RF coil are all placed inside the bore of the EM. A LIA detects the amplitude $(R)$ and phase $(\theta)$ of PL modulation. The LIA is connected to the computer, and $R$ and $\theta$ are recorded along with the position of the battery (the 3D translation stage).

\section{AC Magnetometry}

The PL of a diamond sample displays various cross relaxation features, extensively discussed in the literature [13-15], as a function of the bias magnetic field, as shown in Figure 2. In this paper, we exploit a cross-relaxation feature between NV and P1 centers occurring at $51.2 \mathrm{mT}$ (area B in Figure 2a). This feature is used in the battery measurement because of its improved sensitivity compared to the broad NV-NV cross-relaxation feature near the zero field (area A), and its reduced dependence on the magnetic field compared to cross relaxation with NV centers not aligned along the magnetic field at $60 \mathrm{mT}$ (area C) and to the ground state level anticrossing (GSLAC) feature at $102.4 \mathrm{mT}$ (area D). Figure $2 \mathrm{~b}$ shows the corresponding signal amplitude $R$ detected with the LIA, demodulated at the referenced frequency. To maximize the response to alternating signals, $R$ has to be maximized. The NV features seen in Figure 2 are expected to display a temperature dependence. For example, the NV-P1 cross-relaxation features are expected to shift by $-1.34 \mu \mathrm{T} / \mathrm{K}$ at room temperature [16], which can lead to drifts and be interpreted as magnetic field noise. As noted in the inset (d), we chose to use the features at $52.5 \mathrm{mT}$ to detect the AC magnetic signals. The noise sensitivity of the sensor for the parameters used in this experiment leads to an estimated sensitivity of $40 \mathrm{nT} / \sqrt{\mathrm{Hz}}$ with a bandwidth of $100 \mathrm{kHz}$. It should be pointed out that the bandwidth of this technique can be expanded up to $\mathrm{MHz}$, depending on pump-power intensity and $\mathrm{NV}$-axis alignment with respect to the external magnetic field [11].

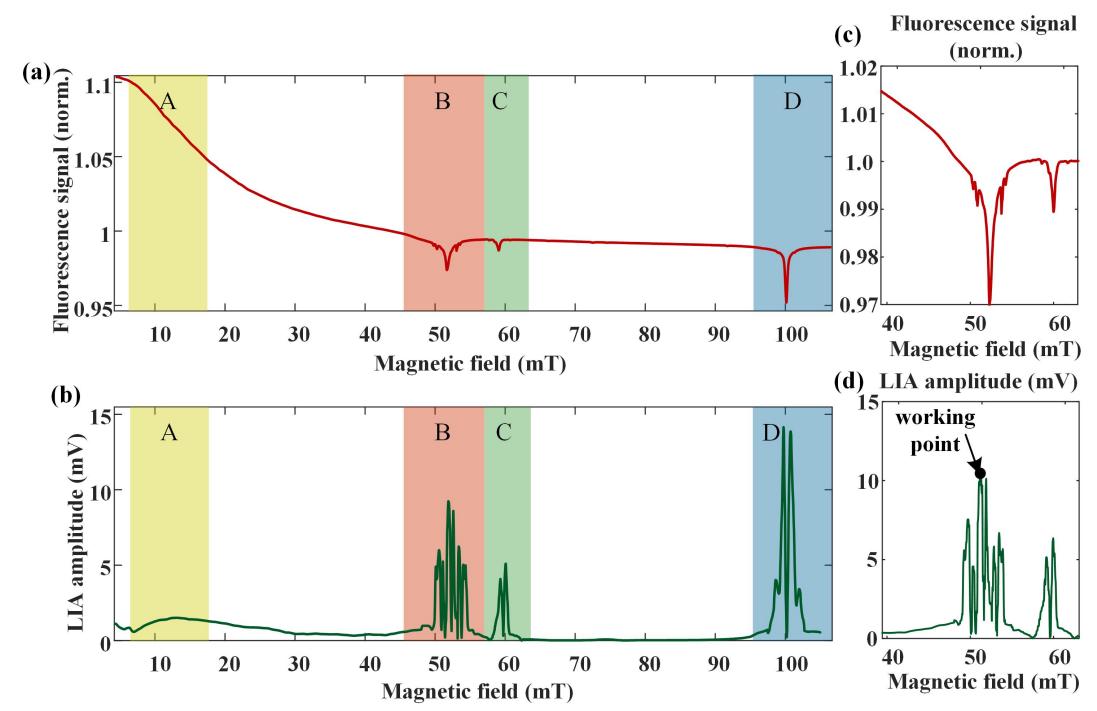

Figure 2. (a) Photoluminescence (PL) of nitrogen-vacancy (NV) centers as a function of the background magnetic field. The four areas A, B, C and D that can be exploited for magnetometry indicate features due to spin mixing, cross relaxation between NV centers and P1 centers, cross relaxation with NV centers that are not aligned along the magnetic field, and ground state level anticrossing, respectively. (b) Corresponding amplitude of lock-in amplifier (LIA) referenced at $100 \mathrm{~Hz}$ for those features. (c) Zoomed-in B area of (a), PL recorded with field-modulation depth of $0.48 \mathrm{mT}$ at $100 \mathrm{~Hz}$. (d) Zoomed-in B area of (b), indicating the working point at $52.5 \mathrm{mT}$. 


\section{Battery Measurement}

The dimensions of the solid state battery measured in this work are $4.0 \times 3.0 \times 1.0 \mathrm{~mm}^{3}$, and are shown in Figure 3a [17]. This comprises Li-based-ceramic-oxide inner electrodes (noted as 2), a Li-based-ceramic-oxide electrolyte (denoted as 3), a copper charge collector (indicated as 1), and external electrodes made of aluminum. Figure $3 \mathrm{~b}$ shows a photograph of the solid battery, which has an artificial $1 \mathrm{~mm}$-length defect at the right external electrode. The battery sample was placed at a distance of $0.1 \mathrm{~mm}$ to the diamond at the perpendicular direction ( $x$-direction), and scanned over the transverse plane ( $y-z$ plane). Figure $3 g-j$ show the spatially resolved phase signals of the LIA at the corresponding modulation frequencies of $1 \mathrm{kHz}, 5 \mathrm{kHz}, 10 \mathrm{kHz}$ and $40 \mathrm{kHz}$, respectively. The external electrodes including the defect are distinctly visible under the modulation frequency of $1 \mathrm{kHz}$ as shown in Figure $3 \mathrm{~g}$. As the modulation frequency increases, the phase $\theta$ shows an image of the battery structure between the electrodes. There the induced field from different portions of the battery can be differentiated, as shown in Figure $3 \mathrm{~h}-\mathrm{j}$. Figure $3 \mathrm{c}-\mathrm{f}$ show the spatially resolved amplitude signals of LIA at the corresponding modulation frequencies of $1 \mathrm{kHz}, 5 \mathrm{kHz}, 10 \mathrm{kHz}$ and $40 \mathrm{kHz}$. In order to investigate the ability of the diamond-based magnetometer to identify structural anomalies inside batteries, we introduced two kinds of artificial anomalies. First, an iron-containing brass cylinder with a height of $1 \mathrm{~mm}$ and diameter of $1 \mathrm{~mm}$ was placed inside the battery, serving as an impurity. The results are shown in Figure $4 a-d$, displaying the spatially resolved amplitude and phase signals of the LIA for the solid battery at frequencies of $5 \mathrm{kHz}$ and $20 \mathrm{kHz}$. This impurity is visible at both frequencies in the phase signal, indicated with red dashed circles in Figure 4. The impurity image is absent in the amplitude signal at frequencies lower than $5 \mathrm{kHz}$, this is because the amplitude variations caused by the impurity, are smaller than the background shifts caused by temperature changes, for the amplitude signal. The other anomaly is made by cutting the battery in half and then attaching the halves back together, leaving no defect visible to the naked eye. The results are shown in Figure 5, where (a)-(d) display spatially resolved amplitude and phase signals of LIA for the solid battery at frequencies of $5 \mathrm{kHz}$ and $20 \mathrm{kHz}$. The crevice is distinctly visible in the middle of the battery in the phase signals at both frequencies, indicated by the yellow ellipses in (b) and (d). In the amplitude signals the crevice is absent again.

The primary field produced at the center of the coil where the battery is located is given by:

$$
B_{\text {primary }}=\frac{\mu_{0} \times N \times I}{2 r_{\text {coil }}},
$$

where $\mu_{0}=4 \pi \times 10^{-7} \mathrm{~T} \times \mathrm{m} / \mathrm{A}$ is the permeability of free space, $N=4$ is the number of turns of the RF coil, $I=0.8 \mathrm{~A}$ is the amplitude of the alternating current through the coil, and $r_{\text {coil }}=4.2 \mathrm{~mm}$ is the radius of the coil. To perform the LIA detection scheme the modulated field amplitude was selected to be $0.48 \mathrm{mT}$, confirmed experimentally by the GSLAC PL scan and theoretically by Equation (1).

For AC measurements, the magnetic susceptibility $\chi_{m}$ yields two quantities $[18,19]$ : the magnitude and phase of the susceptibility. Alternatively, we can express it in the form of complex susceptibility with the real component $\chi_{r}$ and imaginary component $\chi_{i}$,

$$
\chi_{m}=\chi_{r}+i \chi_{i}
$$

where $\chi_{r}$ is the slope of the direct current (DC) magnetization curve $M(H)$ of the battery, and $\chi_{i}$ indicates the dissipative processes in the battery. The secondary field produced by the battery also depends on the skin depth in the conducting material:

$$
\delta=\sqrt{\frac{2}{\sigma \omega \mu}}
$$


(a)
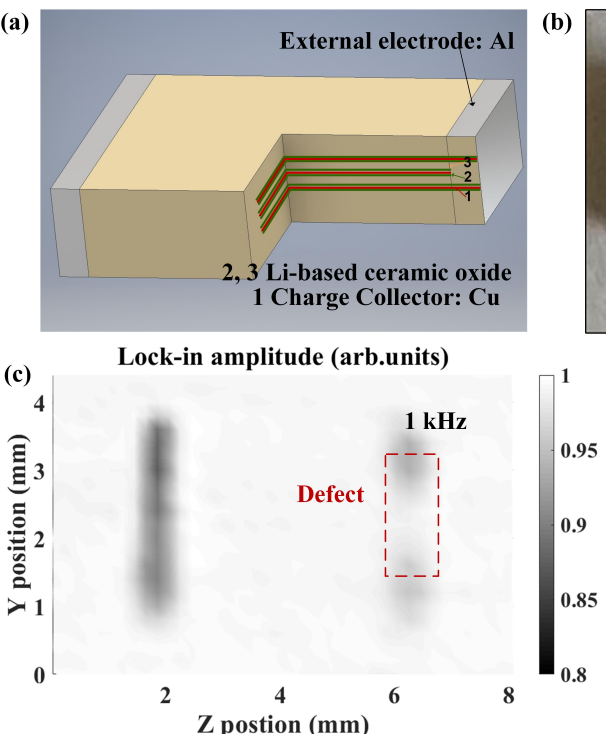

(d) Lock-in amplitude (arb.units)

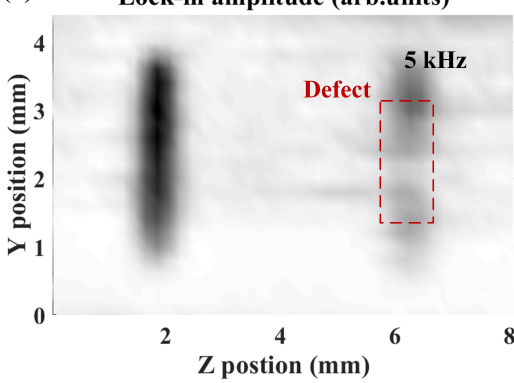

(e) Lock-in amplitude (arb.units)

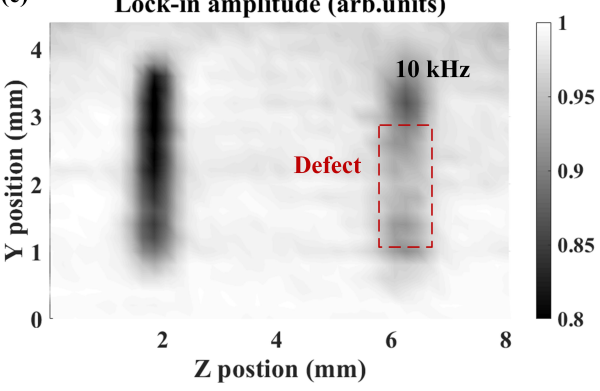

(f) Lock-in amplitude (arb.units)

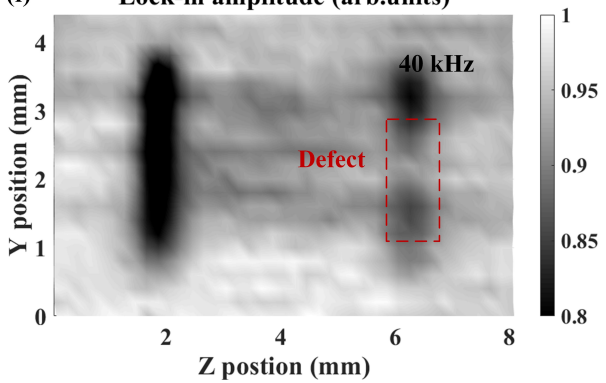

(b)

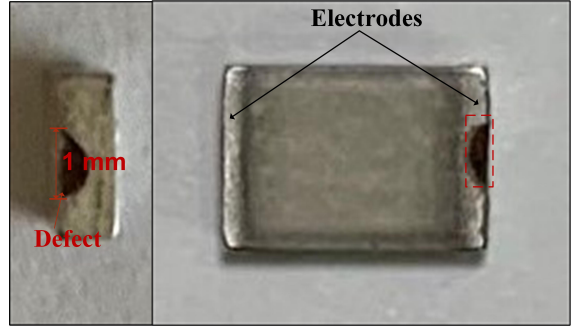

Lock-in phase (arb.units)

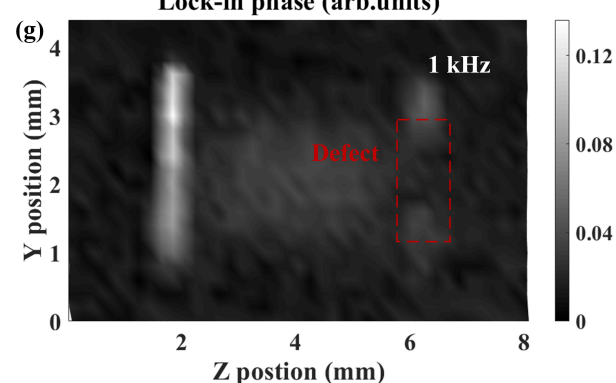

(h) Lock-in phase (arb.units)
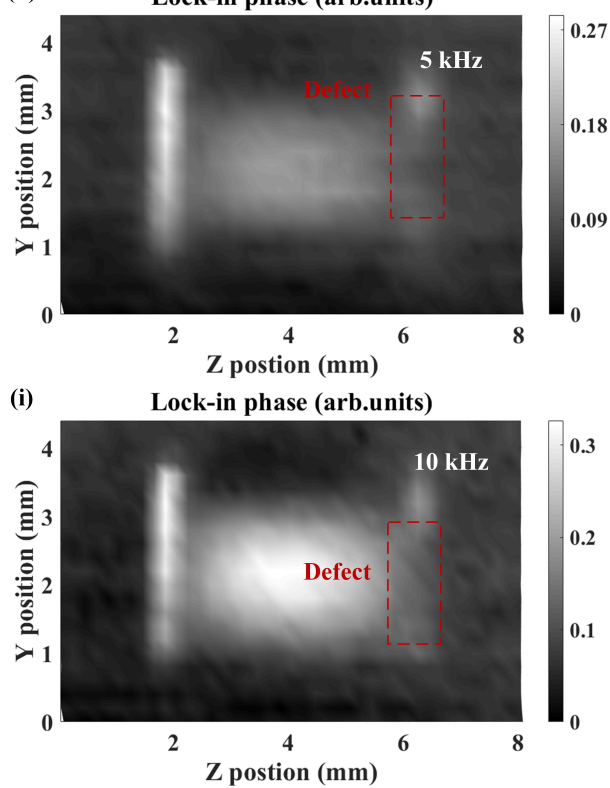

(j) Lock-in phase (arb.units)

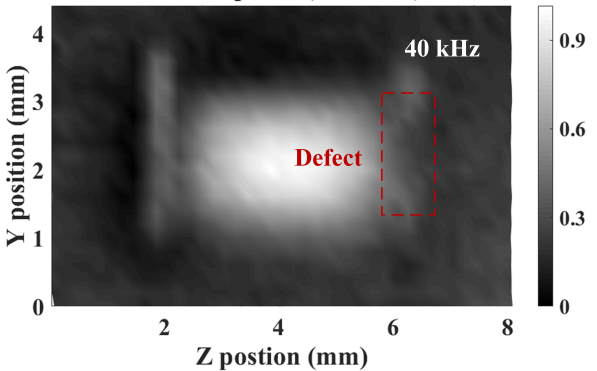

Figure 3. (a) Battery schematic. The ceramic solid battery consists of external aluminum electrodes, Li-based ceramic oxide inner electrodes (noted as 2), a Li-based ceramic oxide electrolyte (noted as 3), and a copper charge collector (indicated as 1). (b) A photograph of the battery with dimension $4 \times 3 \times 1 \mathrm{~mm}^{3}$. Distinctly visible is the defect on the external electrode. (c-f) Maps of the $R$ of LIA for modulation frequencies of $1 \mathrm{kHz}, 5 \mathrm{kHz}, 10 \mathrm{kHz}$ and $40 \mathrm{kHz}$. (g-j) Maps of the $\theta$ of LIA for modulation frequencies of $1 \mathrm{kHz}, 5 \mathrm{kHz}, 10 \mathrm{kHz}$ and $40 \mathrm{kHz}$, respectively. 

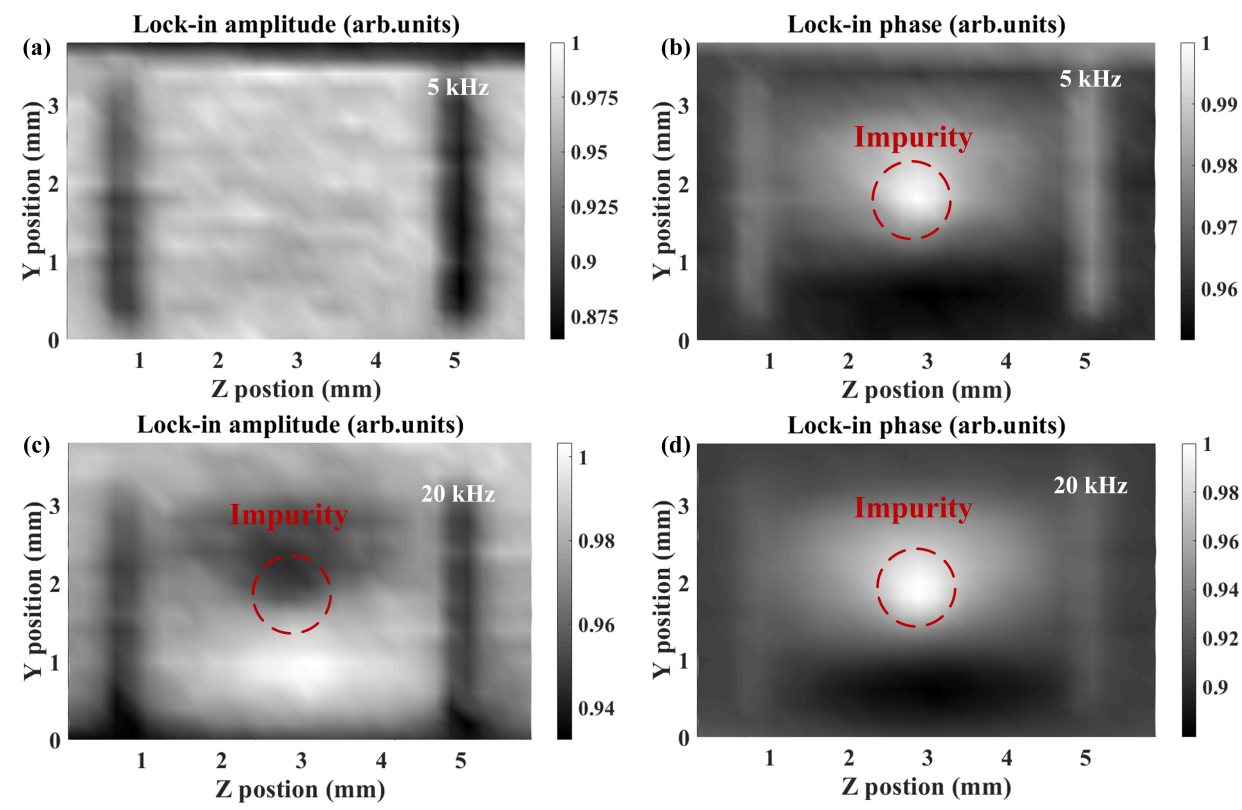

Figure 4. Imaging pictures of solid batteries with the impurity. (a-d) are amplitudes and phases of LIA for the battery with an impurity at frequencies of $5 \mathrm{kHz}$ and $20 \mathrm{kHz}$.
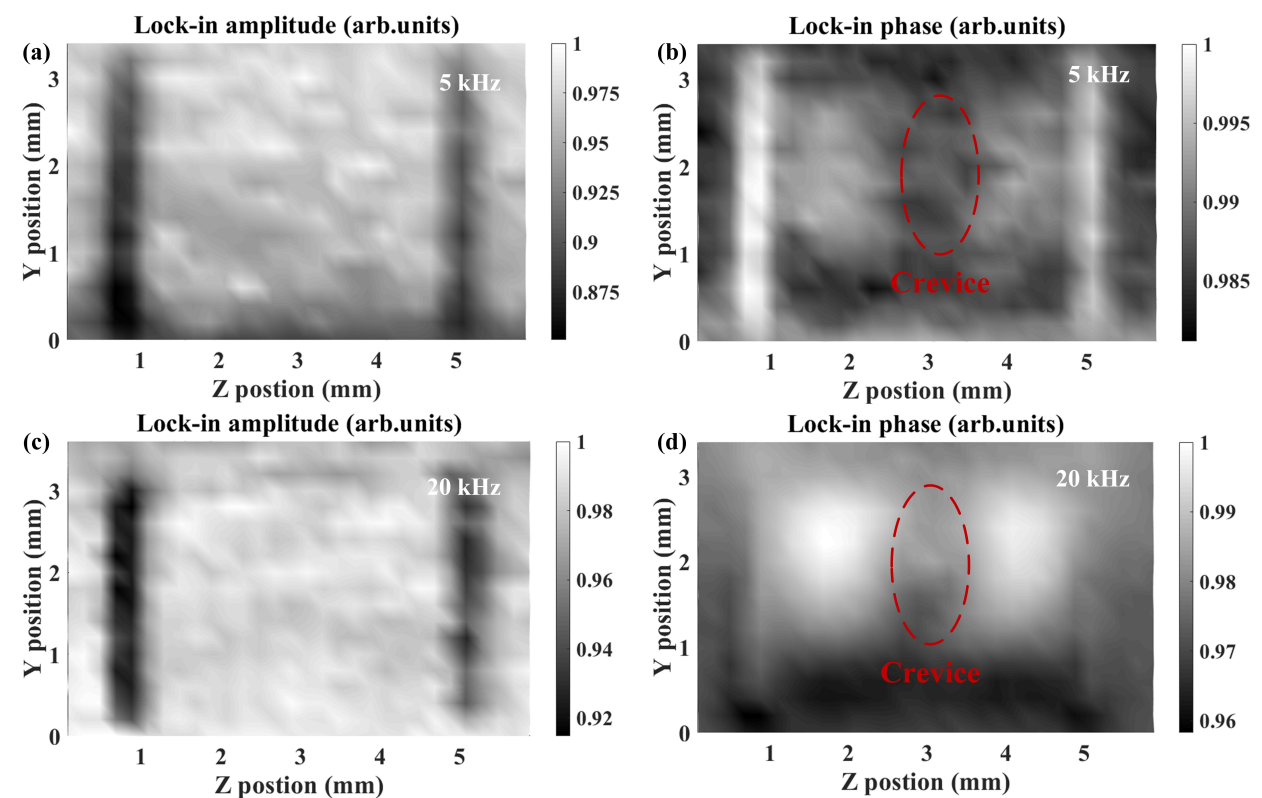

Figure 5. Imaging pictures of solid batteries with the crevice. (a-d) are amplitudes and phases of LIA for the battery with a crevice at frequencies of $5 \mathrm{kHz}$ and $20 \mathrm{kHz}$.

where $\omega=2 \pi f$ denotes the angular frequency, $\sigma$ is the conductivity of the material and $\mu$ is the mutual permeability of the material. In order to estimate the AC magnetic field generated by the battery, we adopted the calibration formula in refernce [8],

$$
\begin{aligned}
& \delta \phi=\arctan \left(\frac{R_{0} \cos \left(\phi_{0}\right)-R_{m} \cos \left(\phi_{m}\right)}{R_{m} \sin \left(\phi_{m}\right)}\right), \\
& \delta R=\sqrt{R_{m}^{2} \sin \left(\phi_{m}\right)^{2}+\left(R_{0} \cos \left(\phi_{0}\right)-R_{m} \cos \left(\phi_{m}\right)\right)^{2}},
\end{aligned}
$$

where $\delta \phi$ indicates the phase shifts generated by the battery, $\delta R$ is the amplitude contrast generated by the battery, $R_{0}$ and $\phi_{0}$ are the background-corresponding amplitude and phase of the LIA, respectively, and $R_{m}$ and $\phi_{m}$ are the measured lock-in amplitude and the phase, respectively. The calibrated results are shown in Figure 6. We calibrated the 
magnetic field and phase shifts generated by the battery (the same as the one used in Figure 3) at a modulation frequency of $5 \mathrm{kHz}$. The external electrode without the defect induces the maximum magnetic field of $0.04 \mathrm{mT}$ and phase shift of $0.03 \mathrm{rad}$.
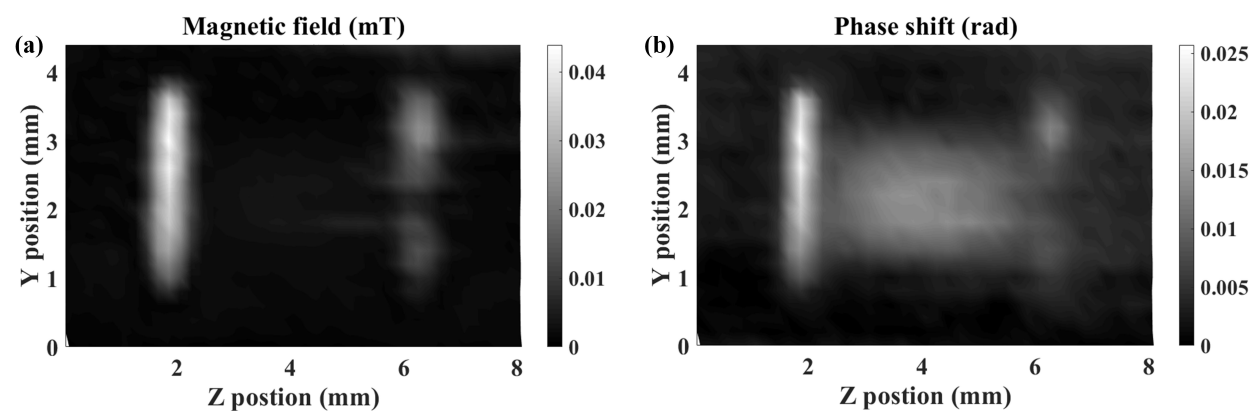

Figure 6. Magnetic field and phase shifts generated by the battery at frequency of $5 \mathrm{kHz}$. (a) Calibrated magnetic field generated by the battery. (b) Phase shifts generated by the battery.

\section{Spatial Resolution}

One of the advantages of diamond-based sensors is their spatial resolution. For determining the spatial resolution of this battery-measurement system, we used data from one of the aluminum-made electrodes of the solid battery. As shown in Figure 7, the average cross section of $0.3 \mathrm{~mm}$-width electrode is expressed by a square function, noted as the green plot, and a Gaussian-function, noted with red, was employed as a kernel to convolve the square function to fit the measured data, denoted as blue dots. All traces have been normalized for better comparison. By adjusting the full width at half-maximum of Gaussian function, we can estimate the spatial resolution of the system. We represent those results with blue. The spatial resolution of the system can be estimated form the full width at half maximum (FWHM) of the kernel and is estimated as $360 \pm 2 \mu \mathrm{m}$. It is mostly restrained by the distance between the battery and diamond. Closer proximity could increase measurement contrast, allowing a more legible image of small samples.

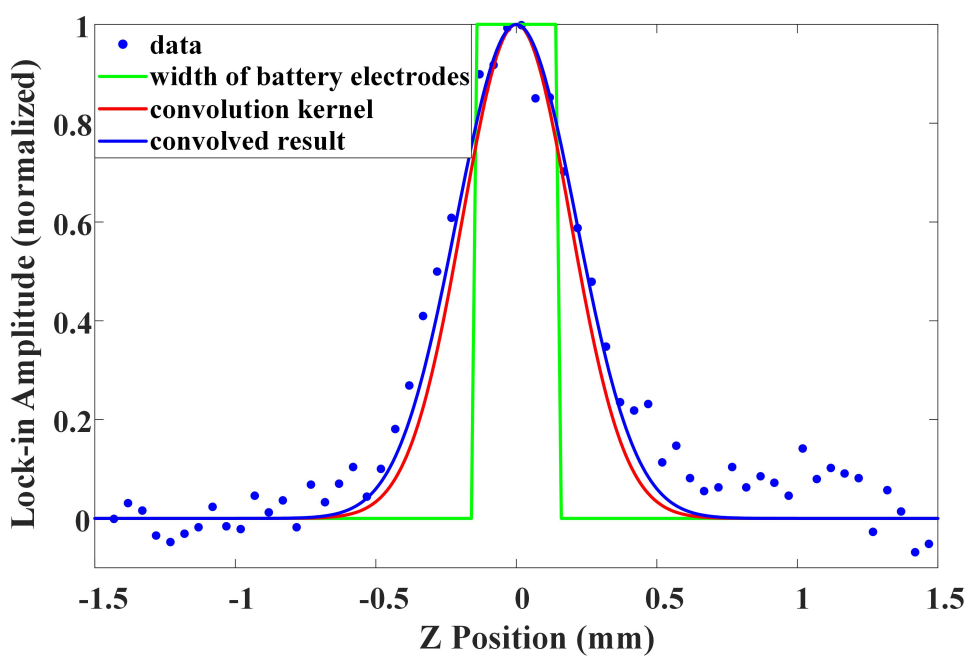

Figure 7. Determination of spatial resolution. The blues dots signify the average cross section of the $0.3 \mathrm{~mm}$-width electrode imaged by the sensor, the green trace denotes the width of electrode of battery as a square function, and the red curve represents a Gaussian function, as a convolution kernel of the green trace to fit the experimental data, noted as a blue trace.

\section{Conclusions and Outlook}

We demonstrated microwave-free eddy-current imaging with NV centers in diamond exploiting the cross-relaxation feature between the NV centers and P1 centers occurring at $51.2 \mathrm{mT}$. The magnetic sensitivity is $40 \mathrm{nT} / \sqrt{\mathrm{Hz}}$ with $100 \mathrm{kHz}$ bandwidth, but as previously 
demonstrated [11], it can be extended to a few MHz. The diamond-based sensor is used to noninvasively image a mm-sized solid battery. The spatially resolved amplitude and phase lock-in signals were used to determine the dimension and shape of the battery features, including a $1 \mathrm{~mm}$-wide artificial defect and $0.3 \mathrm{~mm}$-wide electrodes, from which the spatial resolution was calibrated as $360(2) \mu \mathrm{m}$, which was limited by the distance between the sensor and the battery. Furthermore, the diamond-based probe also identified an anomaly and a crevice inside the battery, demonstrating its ability to detect internal anomalies that are not visible to the naked eye. The maximum magnetic field and phase shift generated by the battery at a modulation frequency of $5 \mathrm{kHz}$ were estimated as $0.04 \mathrm{mT}$ and $0.03 \mathrm{rad}$, respectively.

This paper mainly focuses on testing solid batteries. For future studies, the spatial resolution could be further improved by either using a thinner diamond sample or a diamond with a shallow implanted NV layer to shorten the sensor-sample distance. By tuning the bandwidth, it would be possible to identify structures at different depths inside the battery including electrodes, electrolytes [20] and even material components, which could be useful for battery assessment and development.

Author Contributions: X.Z., G.C. and A.W. conceived the initial idea for the experiment in consultation with D.B., X.Z., G.C. and A.W. designed the experiment with input from all authors. X.Z. and G.C. wrote and implemented control and automation software. X.Z., G.C. and H.Z. constructed the apparatus, performed the experiments, and analyzed the data, with assistance from A.W. and D.B. in data acquisition. All authors discussed results and contributed to the writing of the manuscript. All authors have read and agreed to the published version of the manuscript.

Funding: This work was funded in part by a grant from the U.S. National Science Foundation under award CBET 1804723 and the the German Federal Ministry of Education and Research (BMBF) within the Quantumtechnologien program (FKZ13N14439 and FKZ 13N15064), and by EU FET-OPEN Flagship Project ASTERIQS (action 820394).

Conflicts of Interest: The authors declare no conflict of interest.

\section{References}

1. Wickenbrock, A.; Zheng, H.; Bougas, L.; Leefer, N.; Afach, S.; Jarmola, A.; Acosta, V.M.; Budker, D. Microwave-free magnetometry with nitrogen-vacancy centers in diamond. Appl. Phys. Lett. 2016, 109, 053505. [CrossRef]

2. Kucsko, G.; Maurer, P.C.; Yao, N.Y.; Kubo, M.; Noh, H.J.; Lo, P.K.; Park, H.; Lukin, M.D. Nanometer scale thermometry in a living cell. Nature 2013, 500, 54-58. [CrossRef] [PubMed]

3. Ovartchaiyapong, P.; Lee, K.W.; Myers, B.A.; Jayich, A.C.B.; Stacey, A.; Budker, D.; Hollenberg, L.C.L. Dynamic strain-mediated coupling of a single diamond spin to a mechanical resonator. Nat. Commun. 2014, 5, 4429. [CrossRef] [PubMed]

4. Ajoy, A.; Cappellaro, P. Stable three-axis nuclear-spin gyroscope in diamond. Phys. Rev. A. 2012, 86, 062104. [CrossRef]

5. Ledbetter, M.; Jensen, K.; Fischer, R.; Jarmola, A.; Budker, D. Gyroscopes based on nitrogen-vacancy centers in diamond. Phys. Rev. A 2012, 86, 052116. [CrossRef]

6. Dolde, F.; Fedder, H.; Doherty, M.W.; Nobauer, T.; Rempp, F.; Balasubramanian, G.; Wolf, T.; Reinhard, F.; Hollenberg, L.C.L.; Jelezko, F; et al. Electric-field sensing using single diamond spins. Nat. Phys. 2011, 7, 459-463. [CrossRef]

7. Block, M.; Kobrin, B.; Jarmola, A.; Hsieh, S.; Zu, C.; Figueroa, N.L.; Acosta, V.M.; Minguzzi, J.; Maze, J.R.; Budker, D.; et al. Optically Enhanced Electric Field Sensing using Nitrogen-Vacancy Ensembles. arXiv 2020, arXiv:2004.02886.

8. Wickenbrock, A.; Jurgilas, S.; Dow, A.; Marmugi, L.; Renzoni, R. Magnetic induction tomography using an all-optical 87Rb atomic magnetometer. Opt. Lett. 2014, 39, 6367-6370. [CrossRef] [PubMed]

9. Marmugi, L.; Hussain, S.; Renzoni, F. Electromagnetic induction imaging with a radio-frequency atomic magnetometer. Appl. Phys. Lett. 2016, 108, 103503.

10. Wickenbrock, A.; Leefer, N.; Blanchard, J.W.; Budker, D. Microwave-free magnetometry with nitrogen-vacancy centers in diamond. Appl. Phys. Lett. 2016, 108, 183507. [CrossRef]

11. Chatzidrosos, G.; Wickenbrock, A.; Bougas, L.; Zheng, H.; Tretiak, O.; Yang, Y.; Budker, D. Eddy-current imaging with nitrogen-vacancy centers in diamond. Phys. Rev. Appl. 2019, 11, 014060. [CrossRef]

12. Acosta, V.M.; Bauch, E.; Jarmola, A.; Zipp, L.J.; Ledbetter, M.P.; Budker, D. Broadband magnetometry by infrared-absorption detection of nitrogen-vacancy ensembles in diamond. Appl. Phys. Lett. 2010, 97, 174104. [CrossRef]

13. Zheng, H.; Chatzidrosos, G.; Wickenbrock, A.; Bougas, L.; Lazda, R.; Berzins, A.; Gahbauer, F.H.; Auzinsh, M.; Ferber, R.; Budker, D. Level anti-crossing magnetometry with color centers in diamond. Proc. SPIE 2017, 10119, 101190X. 
14. Hall, L.T.; Kehayias, P.; Simpson, D.A.; Jarmola, A.; Stacey, A.; Budker, D.; Hollenberg, L.C.L. Detection of nanoscale electron spin resonance spectra demonstrated using nitrogen-vacancy centre probes in diamond. Nat. Commun. 2016, 7, 10211. [CrossRef] [PubMed]

15. Anishchik, S.V.; Ivanov, K.L. Sensitive detection of level anticrossing spectra of nitrogen vacancy centers in diamond. Phys. Rev. B 2017, 96, 115142. [CrossRef]

16. Acosta, V.M.; Bauch, E.; Ledbetter, M.P.; Waxman, A.; Bouchard, L.S.; Budker, D. Temperature Dependence of the NitrogenVacancy Magnetic Resonance in Diamond. Phys. Rev. Lett. 2010, 104, 070801. [CrossRef] [PubMed]

17. Hu, Y.; Iwata, G.Z.; Bougas, L.; Blanchard, J.W.; Wickenbrock, A.; Jakob, G.; Schwarz, S.; Schwarzinger, C.; Jerschow, A.; Budker, D. Rapid online solid-state battery diagnostics with optically pumped magnetometers. Appl. Sci. 2020, 10, 7864. [CrossRef]

18. Gomory, F. Complex Harmonic Susceptibilities of Bi2Sr2Ca2Cu3Oy with Different Critical-Current Density. Supercond. Sci. Technol. 1997, 10, 523-542.

19. Mulder, C.A.M.; van Duyneveldt, A.J.; Mydosh, J.A. Susceptibility of the Cu Mn spin-glass: Frequency and field dependences. Phys. Rev. B 1981, 23, 1384. [CrossRef]

20. Hu, Y.; Iwata, G.Z.; Mohammadi, M.; Silletta, E.V.; Wickenbrock, A.; Blanchard, J.W.; Budker, D.; Jerschow, A. Sensitive magnetometry reveals inhomogeneities in charge storage and weak transient internal currents in Li-ion cells. Proc. Natl. Acad. Sci. USA 2020, 117, 10667-10672. [CrossRef] [PubMed] 